\title{
Mixoma em Átrio Esquerdo Associado a Doença Arterial Coronariana Obstrutiva
}

\author{
Left Atrial Myxoma Associated with Obstructive Coronary Artery Disease
}

Ronaldo Altenburg Odebrecht Curi Gismondi, Renato Kaufman, Gabriel Ângelo de Cata Preta Correa, César Nascimento, Luiz Henrique Weitzel, José Oscar Brito Reis, Antônio Sérgio Cordeiro da Rocha, Ademir Batista da Cunha Instituto Nacional de Cardiologia Laranjeiras - Niterói, RJ

\begin{abstract}
Descrevemos o caso de um paciente de 67 anos, portador de doença arterial coronariana obstrutiva, o qual, em avaliação pré-operatória para cirurgia de herniorrafia inguinal, realizou ecocardiograma demonstrando um volumoso tumor em átrio esquerdo, móvel, não-obstrutivo, com pedículo proveniente da veia pulmonar superior direita. O paciente realizou cineangiocoronariografia com ventriculografia esquerda, evidenciando lesão obstrutiva grave em terço médio da artéria descendente anterior, moderada em terço proximal da artéria circunflexa, no local de saída do primeiro ramo marginal, e coronária direita com lesão não-obstrutiva em terço distal. Havia, ainda, disfunção ventricular esquerda moderada. O paciente foi então submetido a cirurgia para retirada do tumor e revascularização do miocárdio. O exame histopatológico mostrou tratar-se de um mixoma.
\end{abstract}

We describe a case of a 67 year-old patient with obstructive coronary artery disease that, in the preoperative survey for inguinal herniorraphy surgery, discovered, by a two-dimensional echocardiogram, a tumor in left atrium, mobile, non-obstructive. The patient underwent a cineangiocoronariography showing severe stenosis in the left anterior descending artery, moderate stenosis in the left circumflex artery, near the origin of the first marginal branch, and a non-obstructive plaque in the right coronary artery. There was also moderate left ventricular dysfunction. After that, the patient has gone coronary artery bypass surgery and resection of the left atrial tumor. The histological exam revealed that the tumor was, in fact, a myxoma.
Os mixomas representam o tipo mais comum entre os tumores cardíacos, correspondendo de $50 \%$ a $60 \%$ do total em algumas casuísticas, com incidência estimada entre 0,5 e um caso por milhão de habitantes-ano ${ }^{1}$. Os sintomas mais comuns incluem dispnéia, dor torácica atípica, fenômenos embólicos e obstrutivos. Já foram descritos casos de morte súbita, provavelmente relacionados com embolização para a circulação coronariana ${ }^{2}$. A presença de doença coronariana concomitante é raramente descrita. O objetivo deste trabalho é relatar o caso de um paciente com mixoma em átrio esquerdo e lesões obstrutivas coronarianas, indicativas de revascularização do miocárdio.

\section{Relato do Caso}

Paciente do sexo masculino, de 67 anos de idade, hipertenso, dislipidêmico, ex-tabagista, com doença pulmonar obstrutiva crônica e passado de infarto agudo do miocárdio em 2000, tratado, na ocasião, com angioplastia sem stent das artérias descendente anterior e coronária direita. Durante avaliação pré-operatória para uma cirurgia de herniorrafia inguinal, realizou ecocardiograma transtorácico, que evidenciou massa tumoral em átrio esquerdo, compatível com mixoma, sendo referido à nossa instituição para abordagem terapêutica.

Na avaliação inicial, o paciente mostrava-se assintomático, com exame físico normal. Eletrocardiograma em ritmo sinusal, com bloqueio completo do ramo esquerdo, hemibloqueio anterior esquerdo, e alterações secundárias da repolarização ventricular. Radiografia de tórax com pulmões hiperinsuflados e área cardíaca normal. O ecocardiograma transesofágico apresentava massa com 4,7 x 1,7 cm de tamanho em átrio esquerdo, móvel, não-obstrutiva ao Doppler, com origem não visualizada, mas parecendo ter pedículo proveniente da veia pulmonar superior direita (fig. 1).

Considerando a história prévia de infarto e a idade do paciente, optou-se pela realização de coronariografia pré-operatória, que mostrou tronco livre de lesões, artéria descendente anterior com lesão grave no terço médio, circunflexa com lesão moderada na saída do primeiro ramo marginal, porém com leito distal fino e irregular, e coronária direita com lesão não-obstrutiva em terço distal (figs. 2 e 3). Havia, ainda, disfunção ventricular esquerda moderada.

O paciente foi submetido a cirurgia para retirada do mixoma em átrio esquerdo e revascularização do miocárdio,

\section{Palavras-chave}

Mixoma, átrio esquerdo, coronariopatia. 


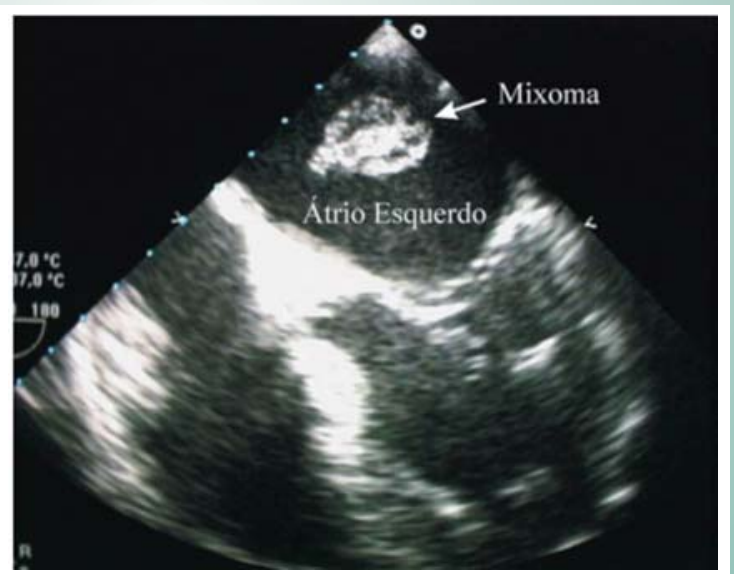

Fig. 1 - Mixoma em átrio esquerdo em imagem bidimensional do ecocardiograma transesofágico.

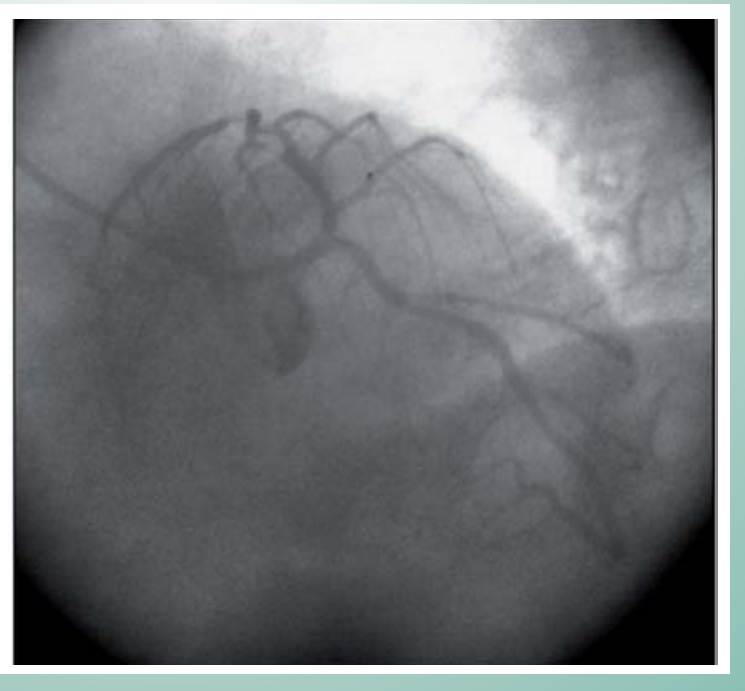

Fig. 3 - Cineangiocoronariogragia em posição craniocaudal (spider), mostrando lesão grave no terço médio da artéria descendente anterior.

com confecção de anastomose entre a artéria torácica interna esquerda e o ramo descendente anterior da artéria coronária esquerda. Evoluiu no pós-operatório sem intercorrências. O material foi enviado para análise histopatológica, que confirmou tratar-se de um mixoma.

\section{Discussão}

O mixoma é uma doença habitualmente sintomática, com a tríade de sintomas emboligênicos, constitucionais inespecíficos ou obstrutivos. A presença de sintomas de doença coronariana nesses pacientes pode ser explicada, em muitos casos, pela embolização de fragmentos do tumor. Não são infreqüentes os relatos de casos de infarto agudo do miocárdio e morte súbita ${ }^{2}$. Todavia, a média de idade alta e a concomitância de outros fatores de risco para aterosclerose tornam a presença de coronariopatia uma importante

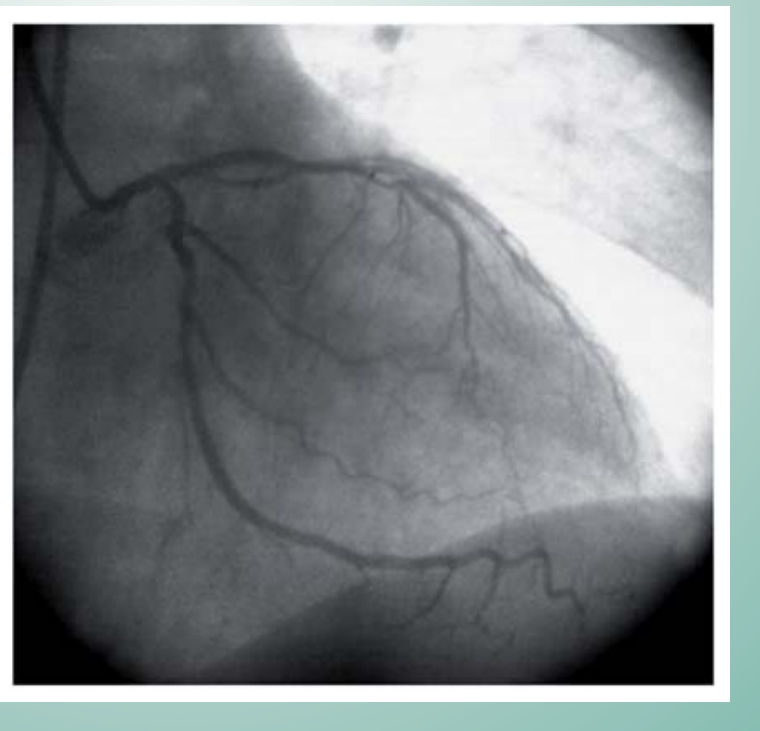

Fig. 2 - Cineangiocoronariografia em posição oblíqua anterior direita, na qual se observa lesão grave no terço médio da artéria descendente anterior.

possibilidade diagnóstica. Há, ainda, autores que defendem um estado de hipercoagulabilidade nos paciente com mixoma, tendo sido mostrado em um relato níveis aumentados de interleucina 6 e $8^{3}$.

A média de idade dos pacientes com mixoma é de 56 anos, e $70 \%$ são do sexo feminino ${ }^{1}$. Algumas publicações anteriores mostravam uma prevalência de doença coronariana em pacientes com mixoma entre 0 e $11 \%^{2}$. Contudo, dois trabalhos atuais mostram valores entre $20,3 \%$ e $36,6 \%$, mais compatíveis com a faixa etária desses pacientes, quando há um maior porcentual de fatores de risco para aterosclerose, como hipertensão arterial sistêmica e dislipidemia ${ }^{1,2}$.

A ecocardiografia continua sendo um excelente exame para diagnóstico e definição topográfica, como tamanho e localização. Cerca de $90 \%$ dos mixomas são solitários e $86 \%$ localizam-se no átrio esquerdo ${ }^{1}$, e o exame por via transesofágica possui maior acurácia que a transtorácica, sendo feito de rotina no pré-operatório em nosso serviço.

Alguns autores defendem que todos os pacientes devam ser submetidos a cirurgia cardíaca tão-logo seja feito o diagnóstico, sem a realização prévia de coronariografia, baseados no risco de morte súbita ${ }^{2,4,5}$. Para outros, esse exame é mandatório apenas nos pacientes com sintomas anginosos ou idade maior que quarenta anos ${ }^{1,2,4}$. Um aspecto também controverso é a ventriculografia, em razão do risco potencial de embolização de fragmentos do tumor que prolapsem do átrio para o ventrículo esquerdo ${ }^{2,4,5}$.

O paciente descrito anteriormente apresentava vários fatores de risco para aterosclerose, como hipertensão arterial sistêmica, tabagismo prévio e história de infarto do miocárdio, tornando imprescindível o estudo hemodinâmico. Além disso, como no ecocardiograma o tumor não apresentava prolapso para o ventrículo, realizou-se a ventriculografia esquerda sem intercorrências. 


\section{Relato de Caso}

Na experiência do nosso serviço é fundamental a realização de coronariografia pré-operatória em todos os pacientes submetidos a cirurgia cardíaca com mais de quarenta anos,

\section{Referências}

1. Erdil N, Ates S, Cetin L, Demirkilic U, Sener E, Tatar H. Frequency of left atrial myxoma with concomitant coronary artery disease. Surg Today. 2003; 33: 328-31.

2. Li AH, Liau CS, Wu CC, Chien KL, Ho YL, Huang CH, et al. Role of coronary angiography in myxoma patients: a 14-year experience in one medical center. Cardiology. 1999; 92: 232-5.

3. Isobe N, Kanda T, Sakamoto H, Morishita Y, Suzuki T, Kobayashi I. Myocardial história prévia de doença coronariana ou sintomas anginosos. A técnica cirúrgica utilizada é a mesma para as cirurgias convencionais de revascularização do miocárdio. infarction in myxoma patients with normal coronary arteries. Angiology. 1996; 47: 819-23.

4. Rice PL, Pifarre R. Left atrial myxoma and coronary artery disease: combined surgical treatment. Arch Surg. 1981; 116: 353-5.

5. Van Cleemput J, Daenen W, De Geest H. Coronary angiography in cardiac myxomas: findings in 19 consecutive cases and review of the literature. Cathet Cardiovasc Diagn. 1993; 29: 217-20. 